\title{
Beauty Production and Identification at CMS
}

Alexander Schmidt

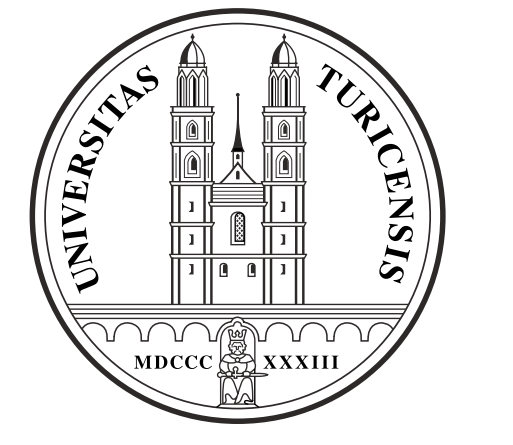

Universität Zürich

Physik-Institut

presented at:

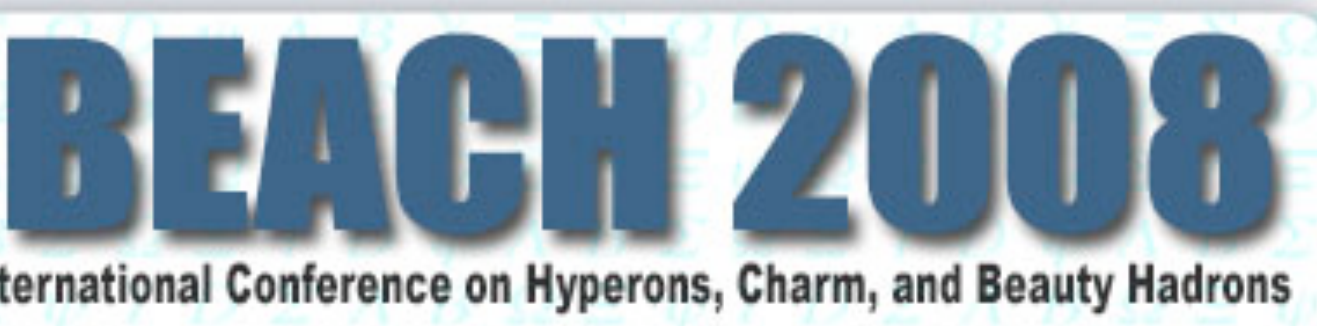

The 8th International Conference on Hyperons, Charm, and Beauty Hadrons

22. 28 JTWOE 2008 UNIVERSITY OF SOUTH CAROLINA 


\section{Outline}

- the CMS detector

- b-production in hadron collisions

- applications of b-identification methods

- some technical details about b-tagging at CMS and expected performance

- prospects for inclusive b-production measurement

- further methods of measuring flavour fractions in jets 


\section{CMS Detector}

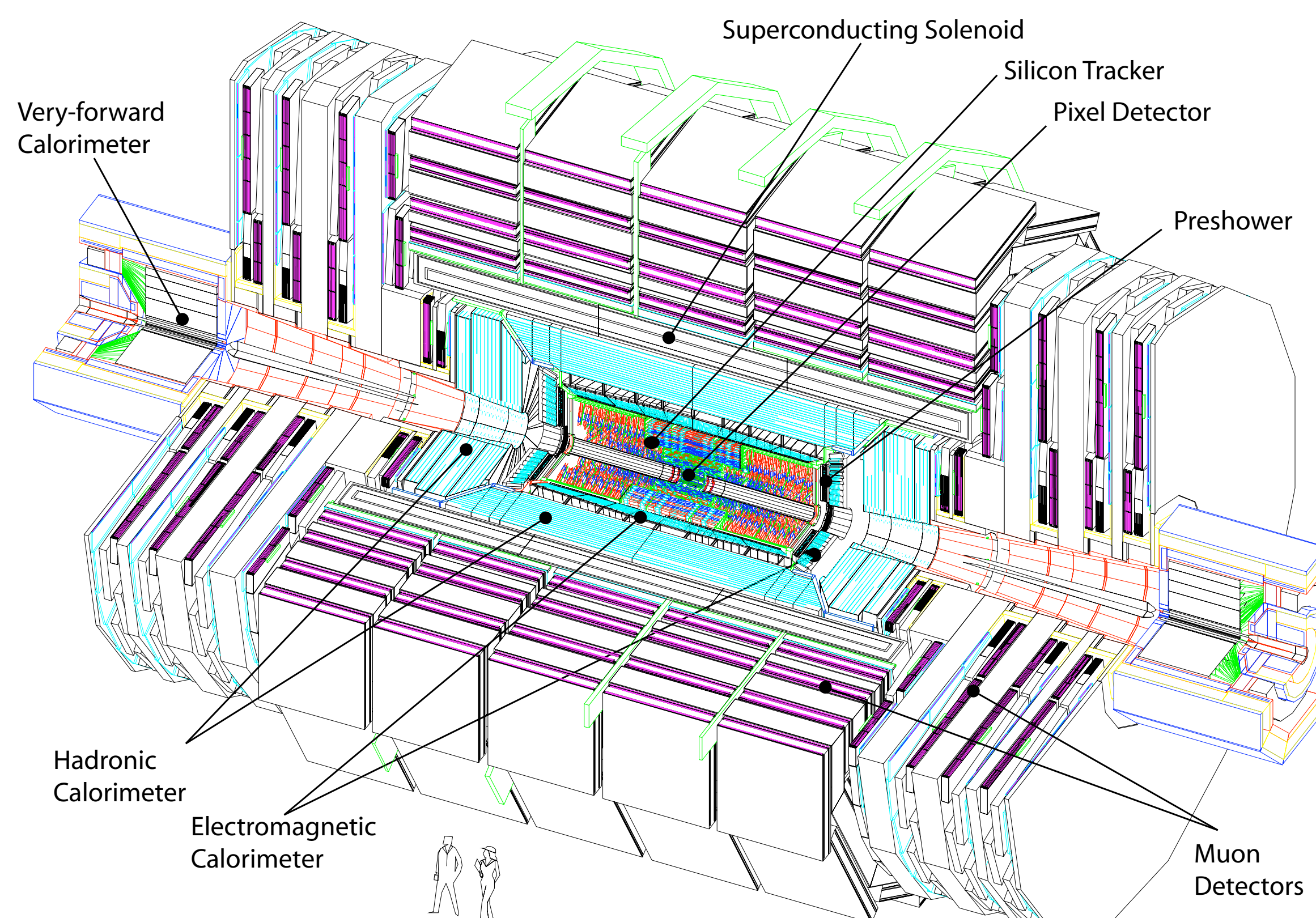

Compact Muon Solenoid magnet:

4 Tesla superconducting solenoid successfully tested

calorimeters: ECAL: 76000 crystals of $\mathrm{PbWO}_{4}$

HCAL: brass absorber + scintillator sandwich

muon system: barrel: drift tubes endcap: cathode strip chambers barrel+endcap: resistive plate chambers

trigger:

$40 \mathrm{MHz}$ bunch crossing rate reduced to $100 \mathrm{~Hz}$ by Level- I and High Level Triggers 


\section{Further Sources of $b$ Quarks}

b quarks play a crucial role in the search for new physics at LHC:

\section{Standard Model Higgs Boson:}

- decays into $b \bar{b}$ for low Higgs masses (B.R. > 50\%)

- candidate channel: association with top - suffers from $\bar{t} \bar{t}+$ jets backgrounds

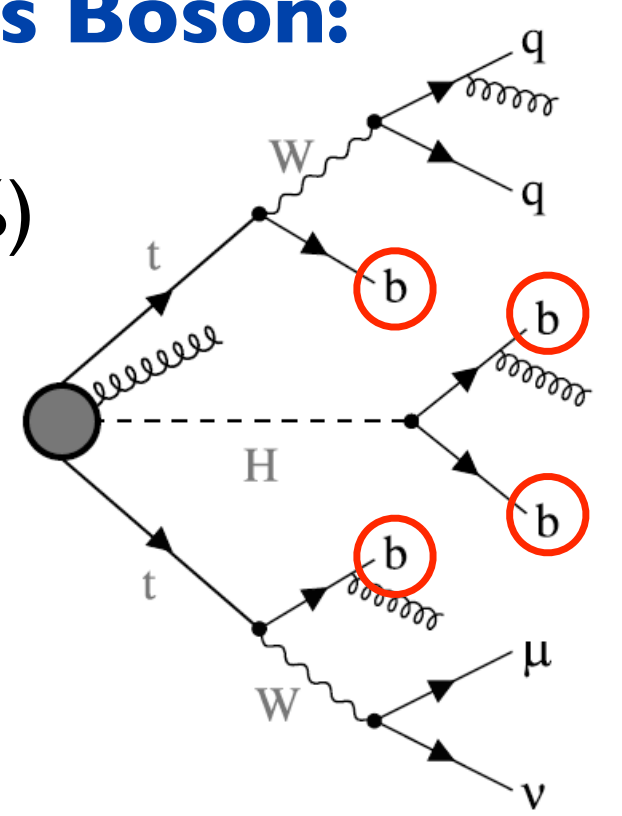

Higgs J. Phys. G: Nucl. Part. Phys. 34 (2007) N221-N250 I
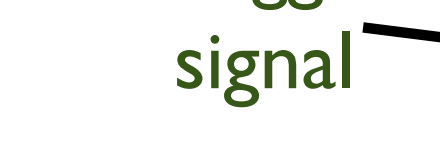

background from misidentified jets

\footnotetext{
$=>$ more work needs to
} be done in this case

\section{SUSY decay chains lead to} final sates with $\mathbf{b} \bar{b}$ :

example $h^{0}$ at LM5:
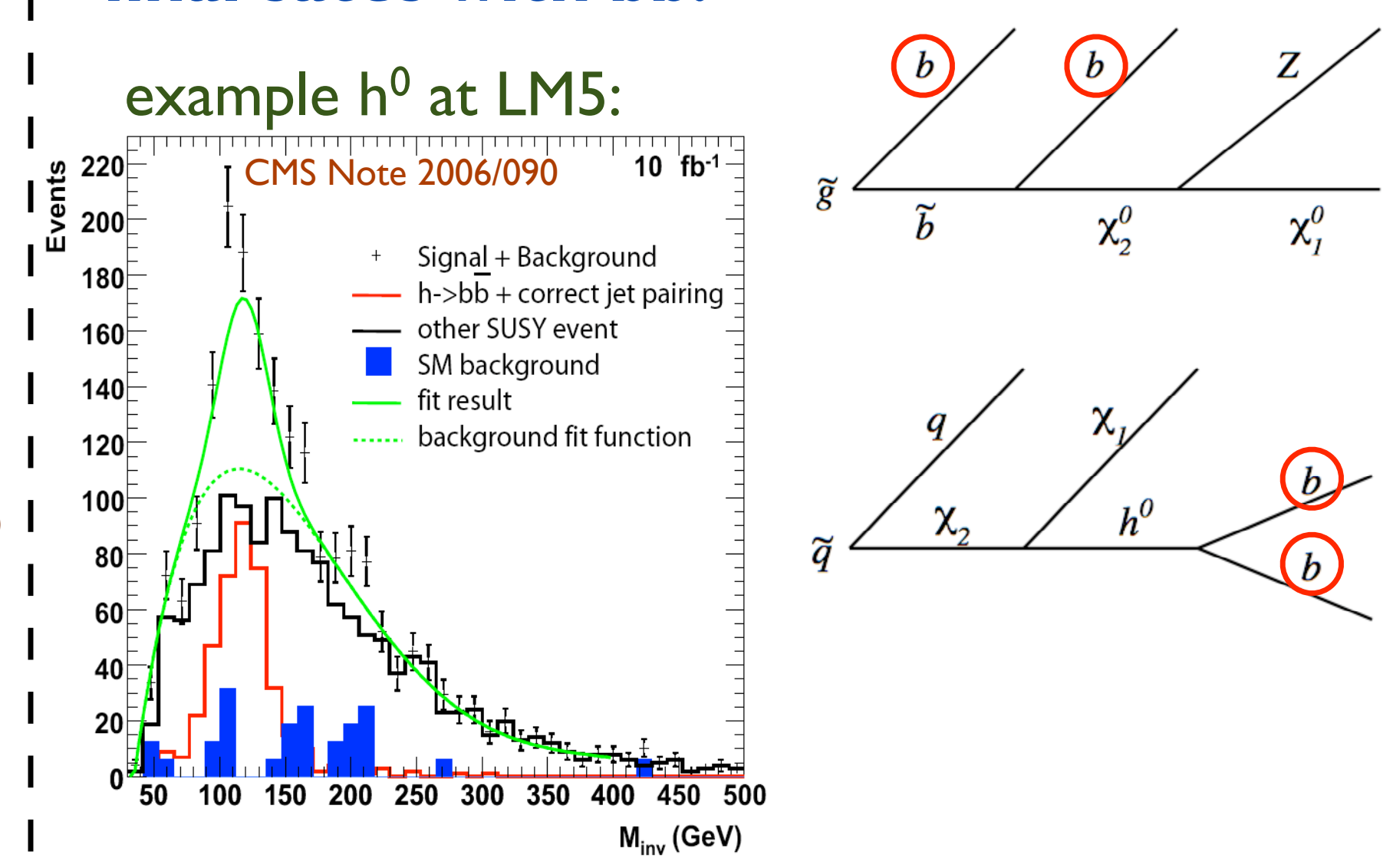

an accurate knowledge of the $\mathrm{b} \overline{\mathrm{b}}$ production rate is critical for an understanding of backgrounds in many BSM searches 


\section{Observables used for $b$-Tagging}

\section{goal: identify b-decay in jet as efficient as possible}

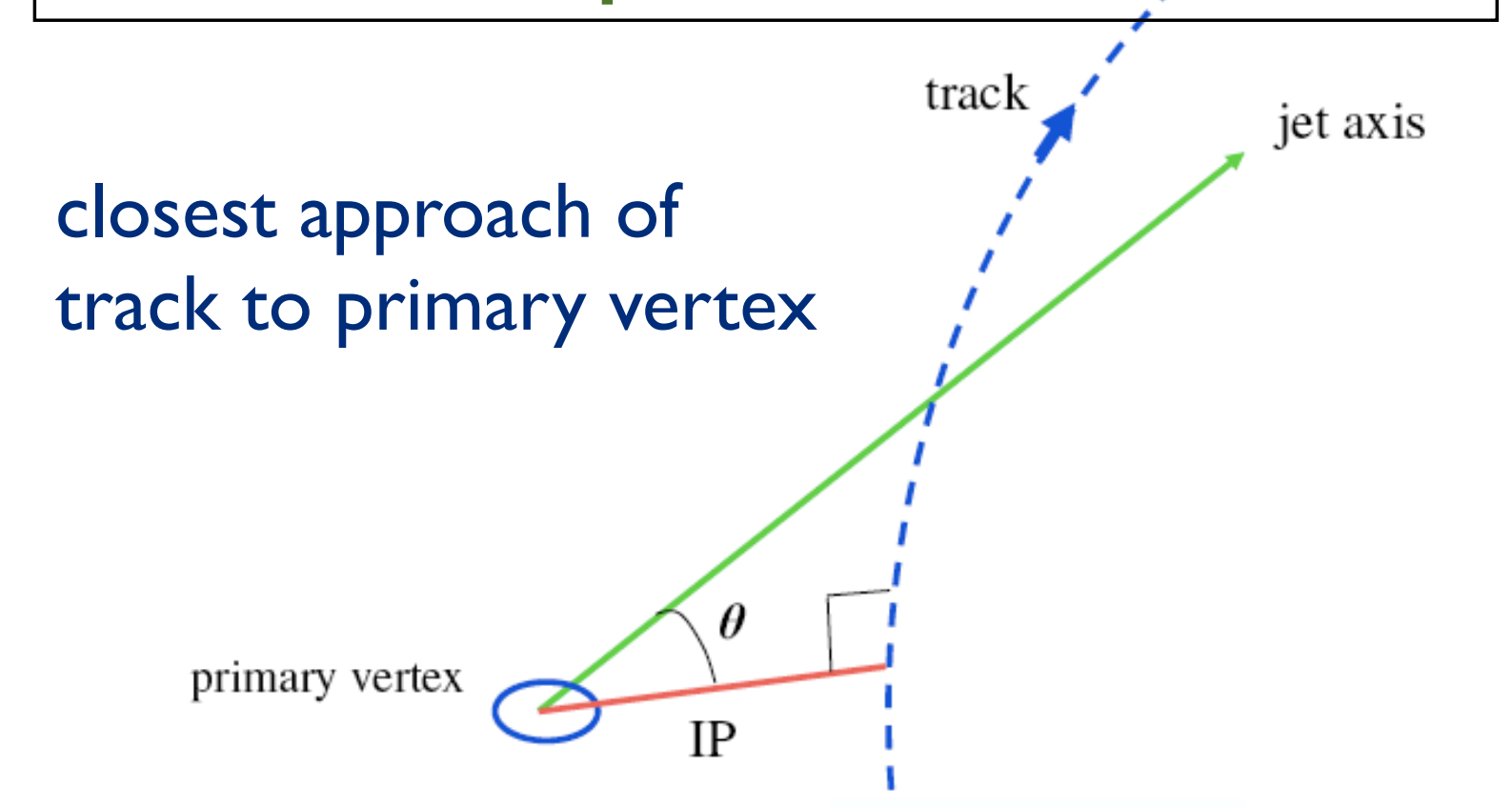

positive (negative) if decay occurs upstream (downstream) the jet direction.

other observables based on secondary vertex:

- flight distance (separation from primary vertex)

- invariant mass at vertex (large b mass compared to $c$ )

- number of tracks at vertex (on average 5 for b)

$-\ldots$.

include all available information into combined tagging algorithms based on Likelihood methods or neural networks

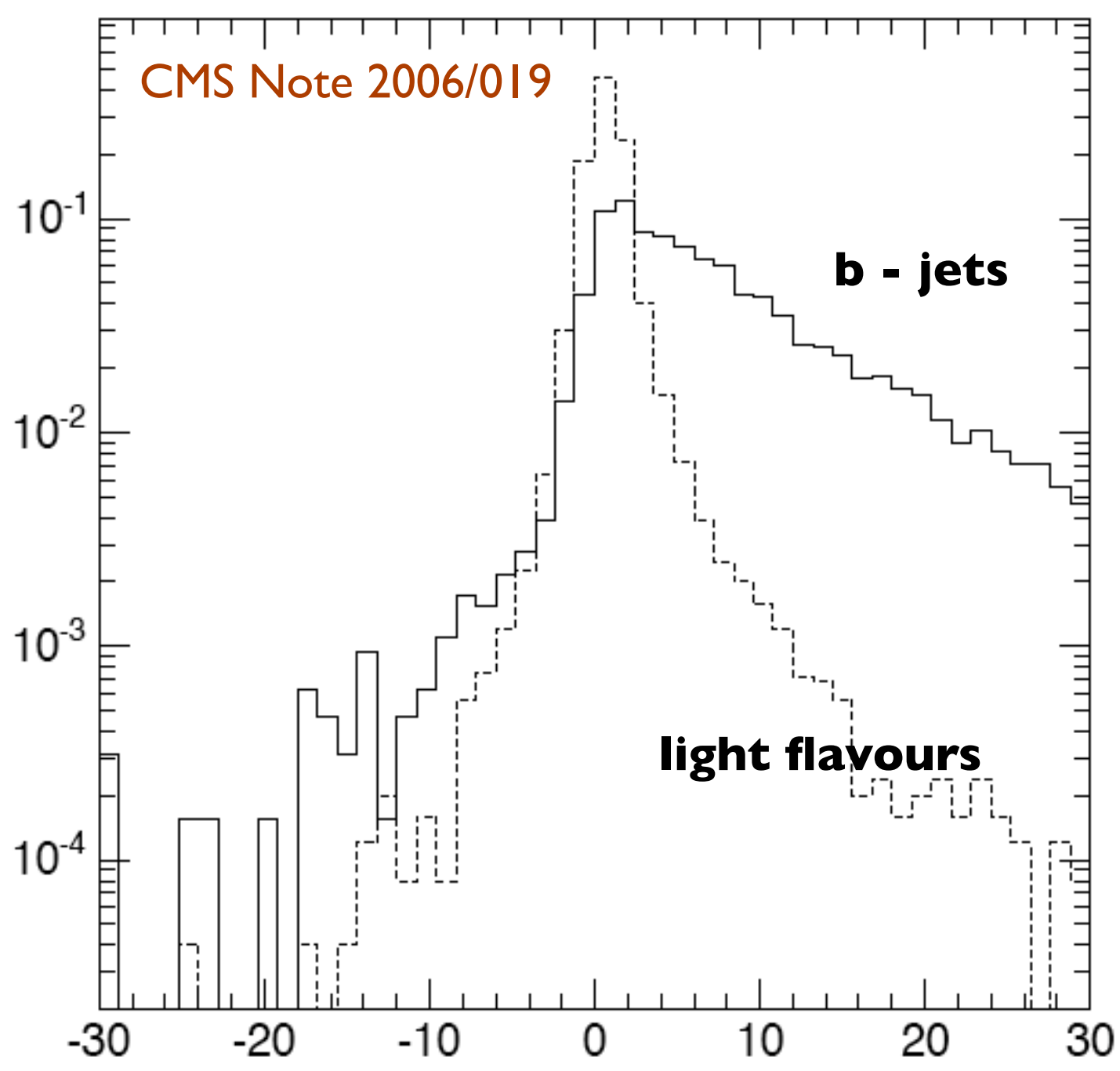

Impact Parameter Significance of 2nd track

$=>$ using significance of second track (ordered by the significance itself) because first track is likely to be mismeasured.

second track $=>$ higher efficiency third track => higher purity 


\section{Detector Alignment}

the achievable performance strongly depends on detector alignment and calibration. scenarios under study:

- startup: installation precision + survey + cosmics (e.g. $20 \mu \mathrm{m}$ for pixel barrel sensors)

- first collisions: I pb $^{-1}:$ using low mass resonances like J/psi and Upsilon improvement by a factor of $\sim 5$ for pixel detector

- I00pb-1: using high mass resonances like $W$ and $Z$

- I $000 \mathrm{pb}^{-1}$ : tracker can be considered to be aligned

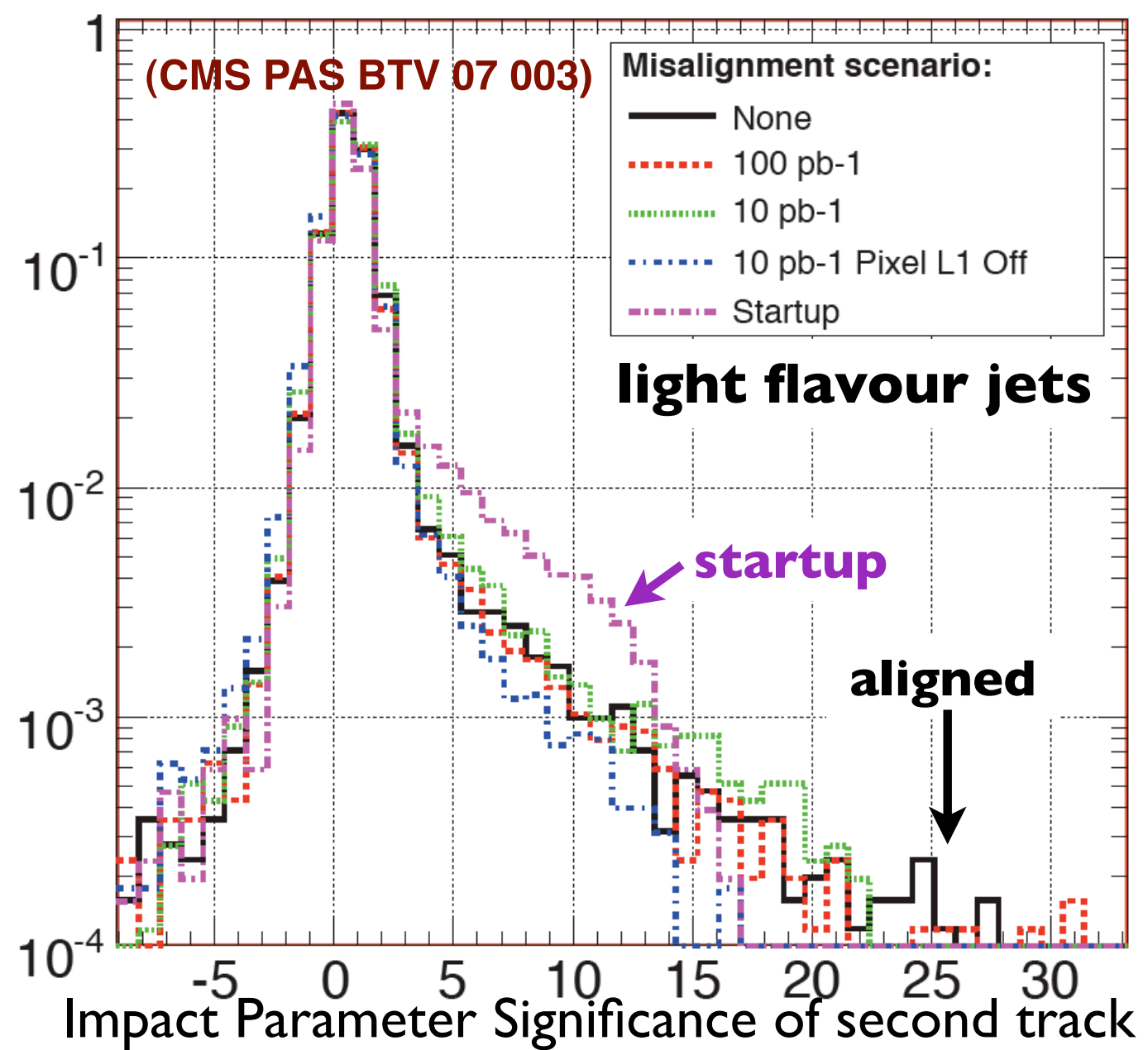

$=>$ broader for startup due to large fake track rate (IP value larger)

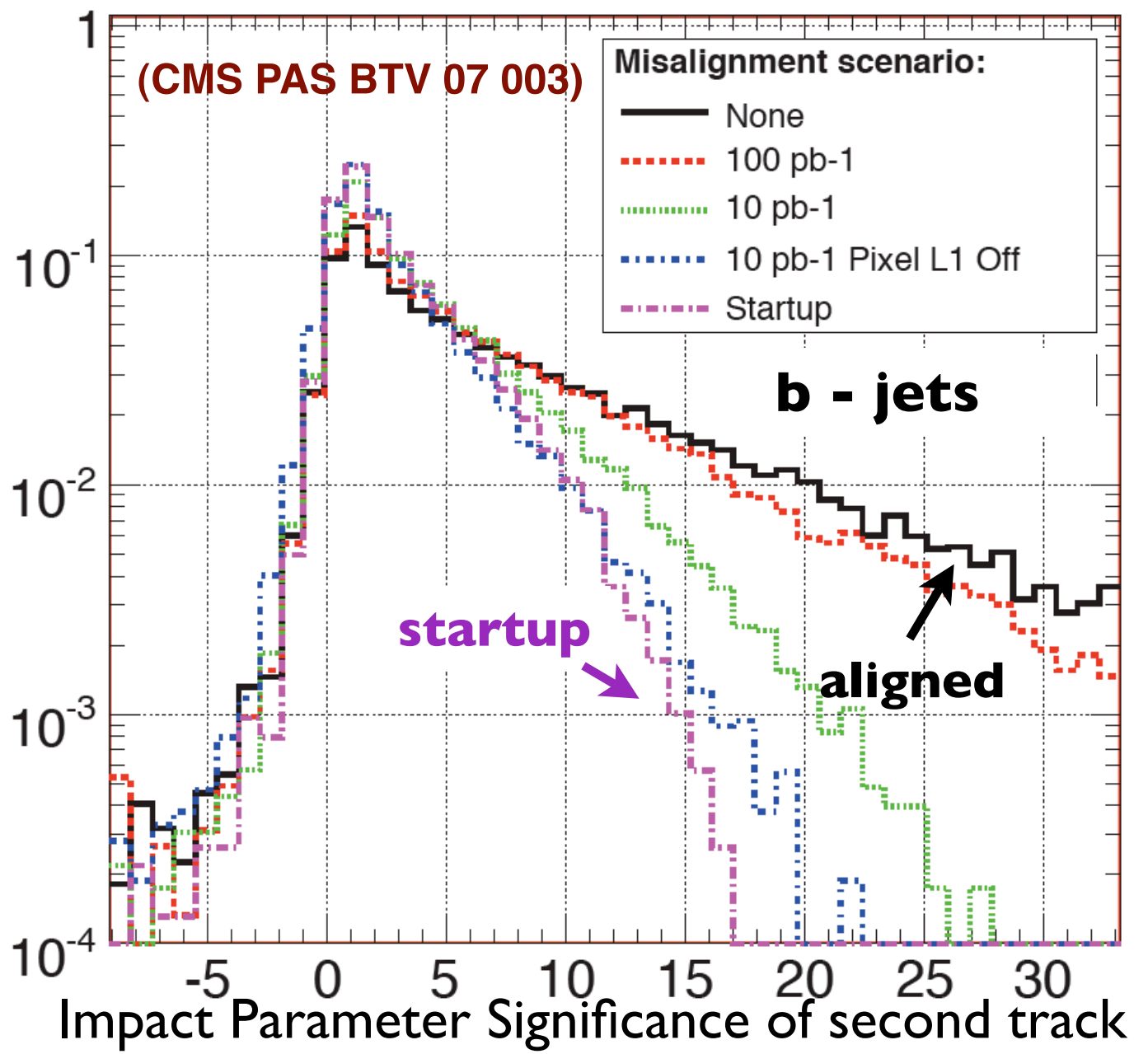

$=>$ more narrow for startup due to large measurement errors (IP error larger) 


\section{Performance of b-Tagging (I)}

example: impact parameter based b-tagging

(using second track, ordered by IP)

- very simple, does not require training of any kind (likelihood, NN) $=>$ application with first data is straightforward

- still reaches high purities

- but it is quite sensitive to misalignment

light flavour misidentification:

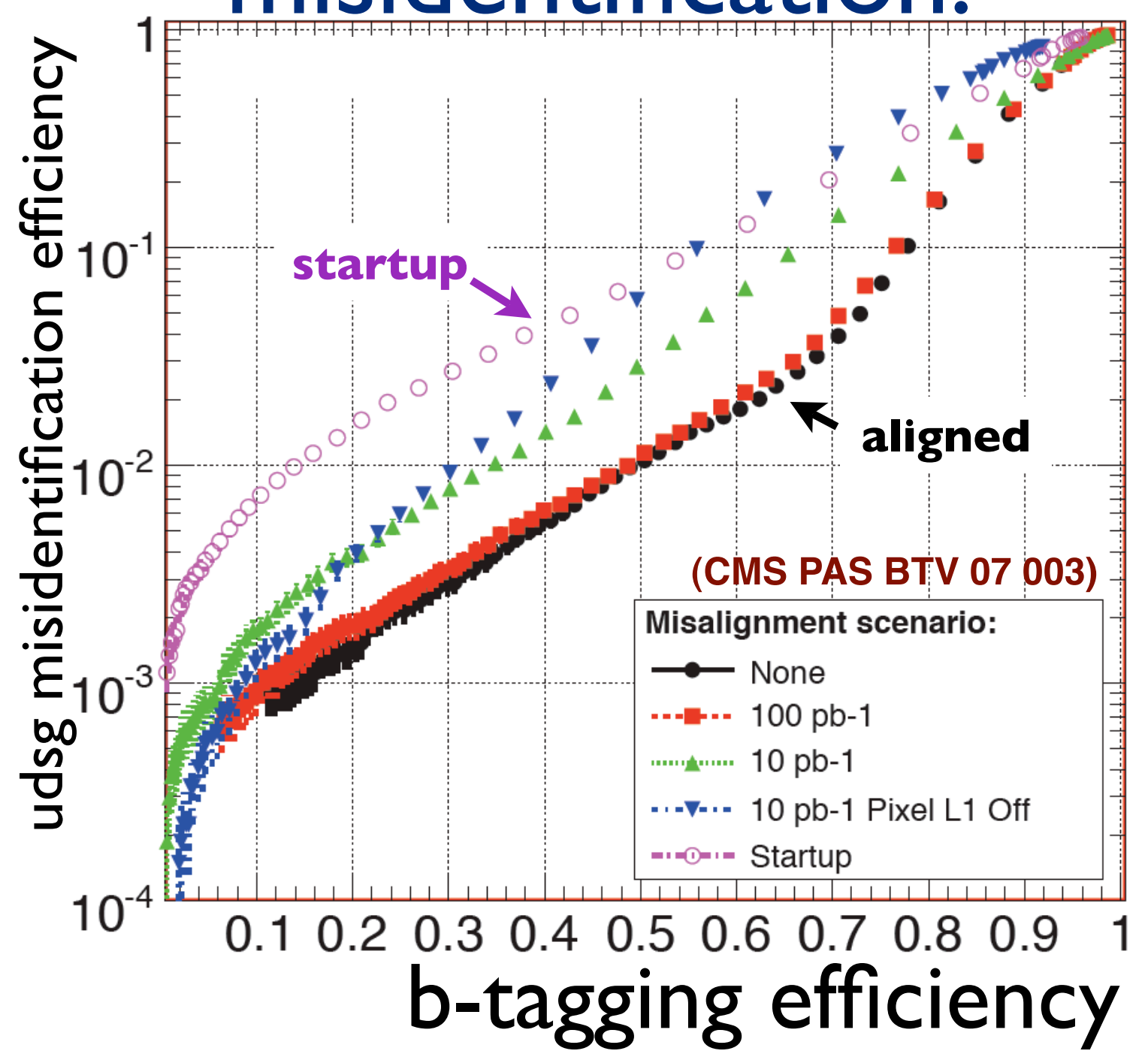

charm misidentification:

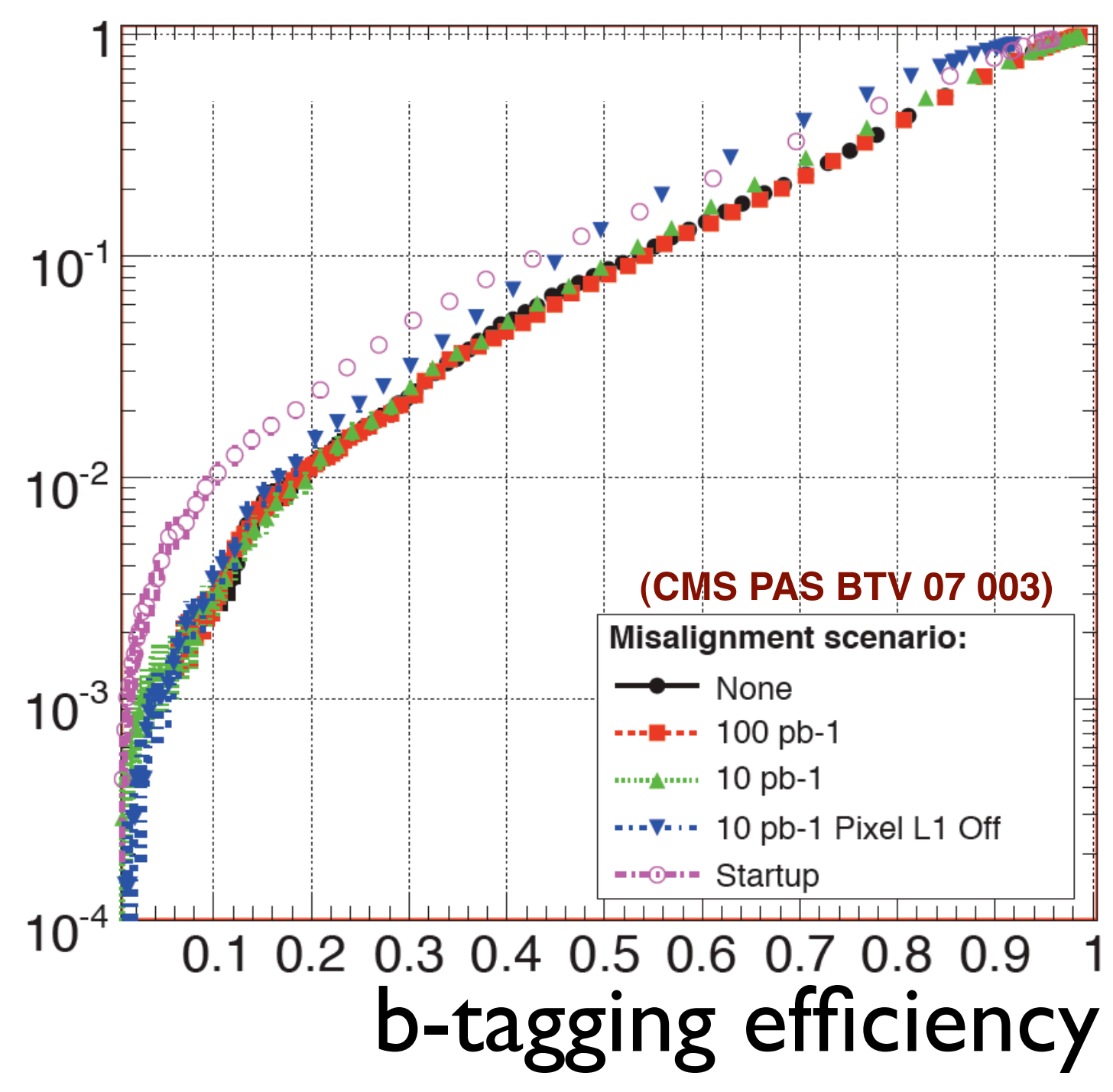

Alexander Schmidt, Universität Zürich

BEACH08, University of South Carolina

June 27th, 2008 


\section{Performance of b-Tagging (II)}

\section{secondary vertex based b-tagging, two examples:}

A. secondary vertex only,

using flight distance significance:

- also very simple, no training

- still reaches high purities

- turns out to be robust against misalignment

- limited to SV finding efficiency $=>2 \%$ mis-tags at $65 \%$ b-efficiency (startup: $3 \%$ mis-tags at $35 \%$ b-efficiency) decrease of performance with misalignment for various algorithms:

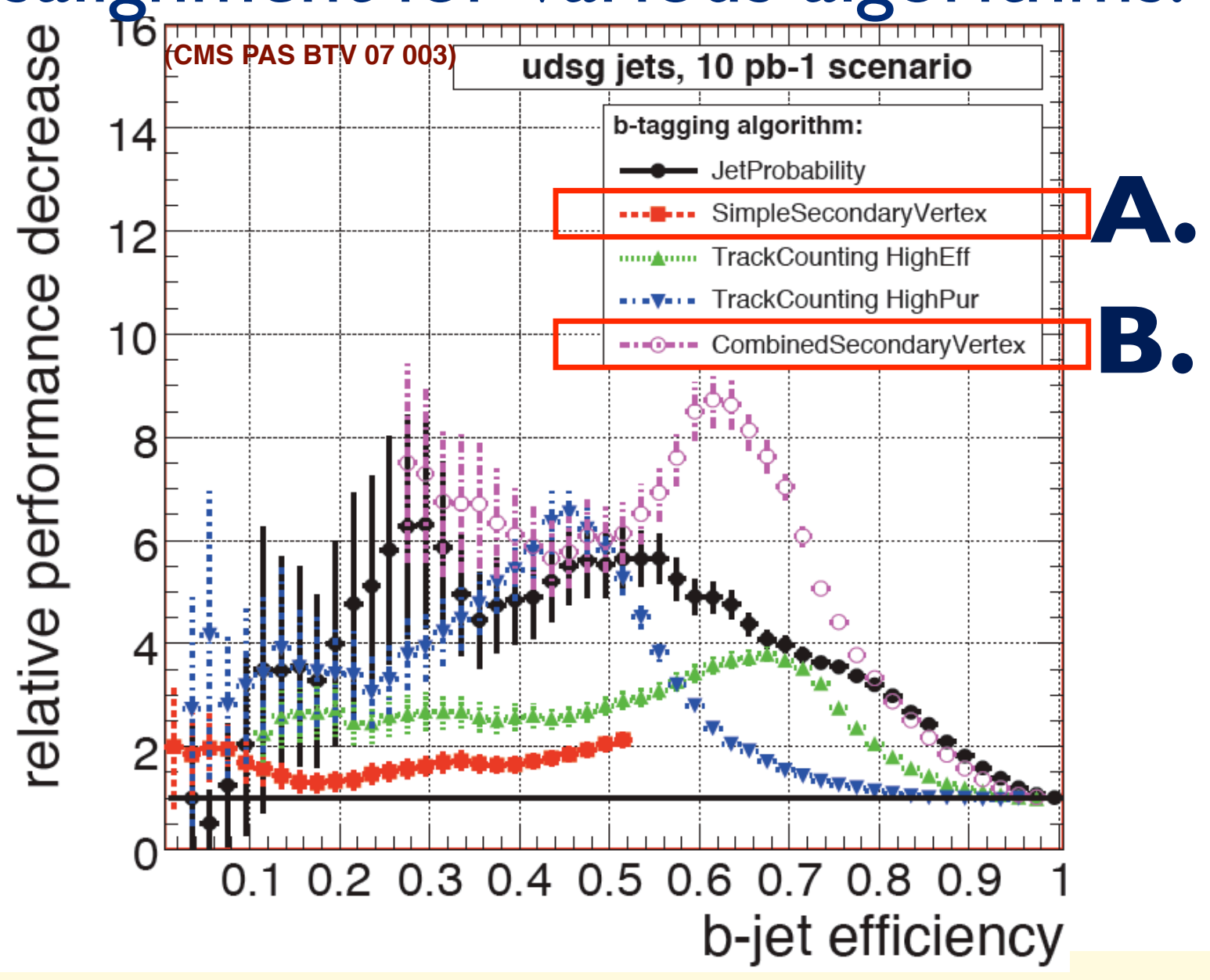

Alexander Schmidt, Universität Zürich
B. combined algorithm

- combining all available information $=>$ most performant algorithm

- requires training

- strongly affected by misalignment

light flavour misidentification for combined algorithm:

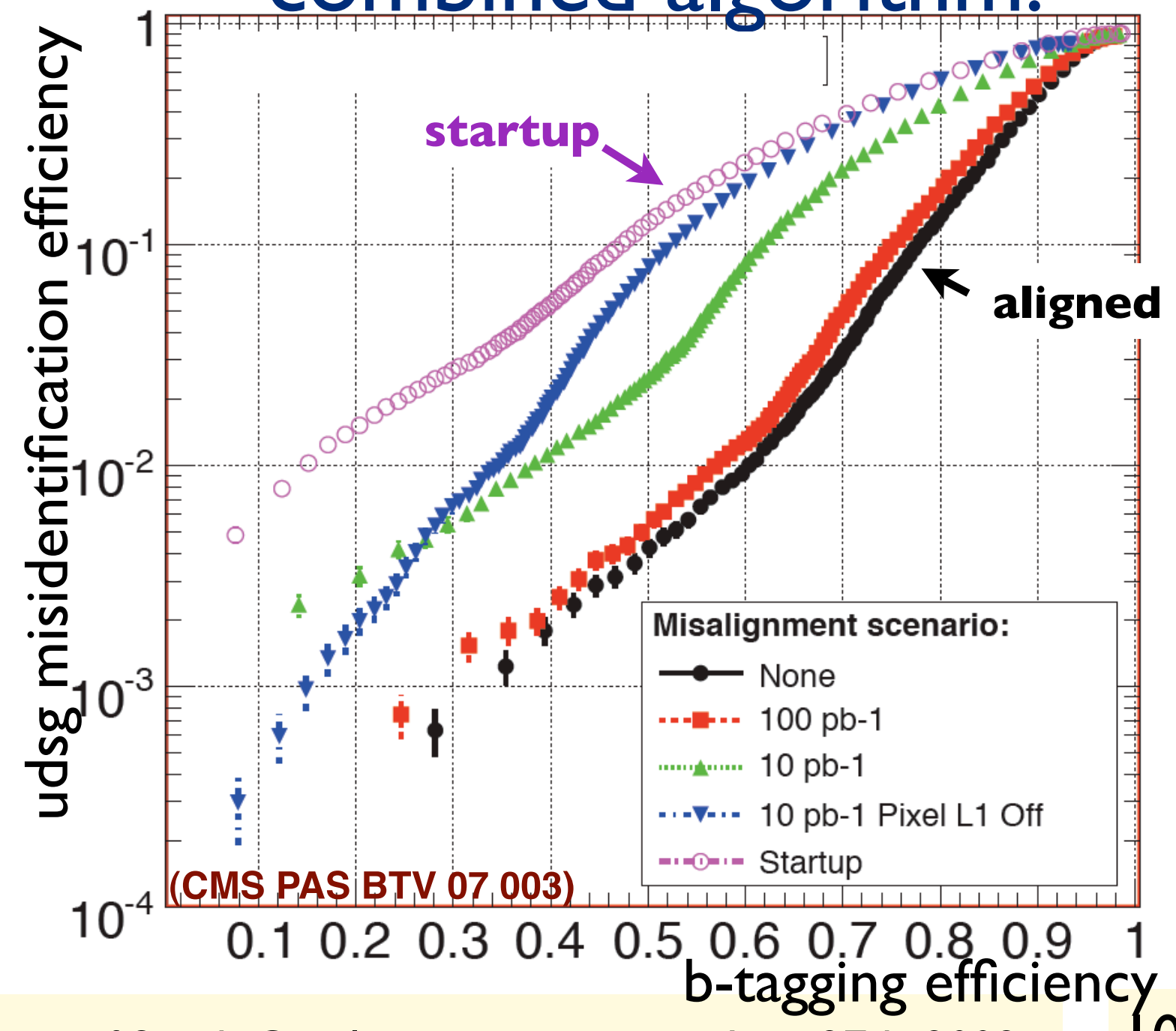




\section{Measurement of Inclusive b-Production (I)}

study of differential cross sections of incl. b-production in

a full detector simulation:

- generated event samples for the simulation :

QCD jets with PYTHIA containing 6\% b-jets t⿱亠幺 production as background $\sigma=500 \mathrm{pb}$

- Trigger: using a muon+b-jet trigger giving

\begin{tabular}{|c|c|}
\hline$\hat{p}_{\mathrm{T}}$, & $\sigma^{\mathrm{QCD}}$, \\
$\mathrm{GeV} / c$ & $\mu \mathrm{b}$ \\
\hline $50-80$ & 20.9 \\
$80-120$ & 3.0 \\
$120-170$ & 0.5 \\
$170-230$ & 0.1 \\
$230-300$ & $2.4 \times 10^{-2}$ \\
$300-380$ & $6.4 \times 10^{-3}$ \\
$380-470$ & $1.9 \times 10^{-3}$ \\
$470-600$ & $6.9 \times 10^{-4}$ \\
$600-800$ & $2.0 \times 10^{-4}$ \\
$800-1000$ & $3.6 \times 10^{-5}$ \\
$1000-1400$ & $1.1 \times 10^{-5}$ \\
\hline
\end{tabular}

a total event rate of $6.1 \mathrm{~Hz}$ at $\mathcal{L}=2 \times 10^{33} \mathrm{~cm}^{-2} \mathrm{~s}^{-1}$

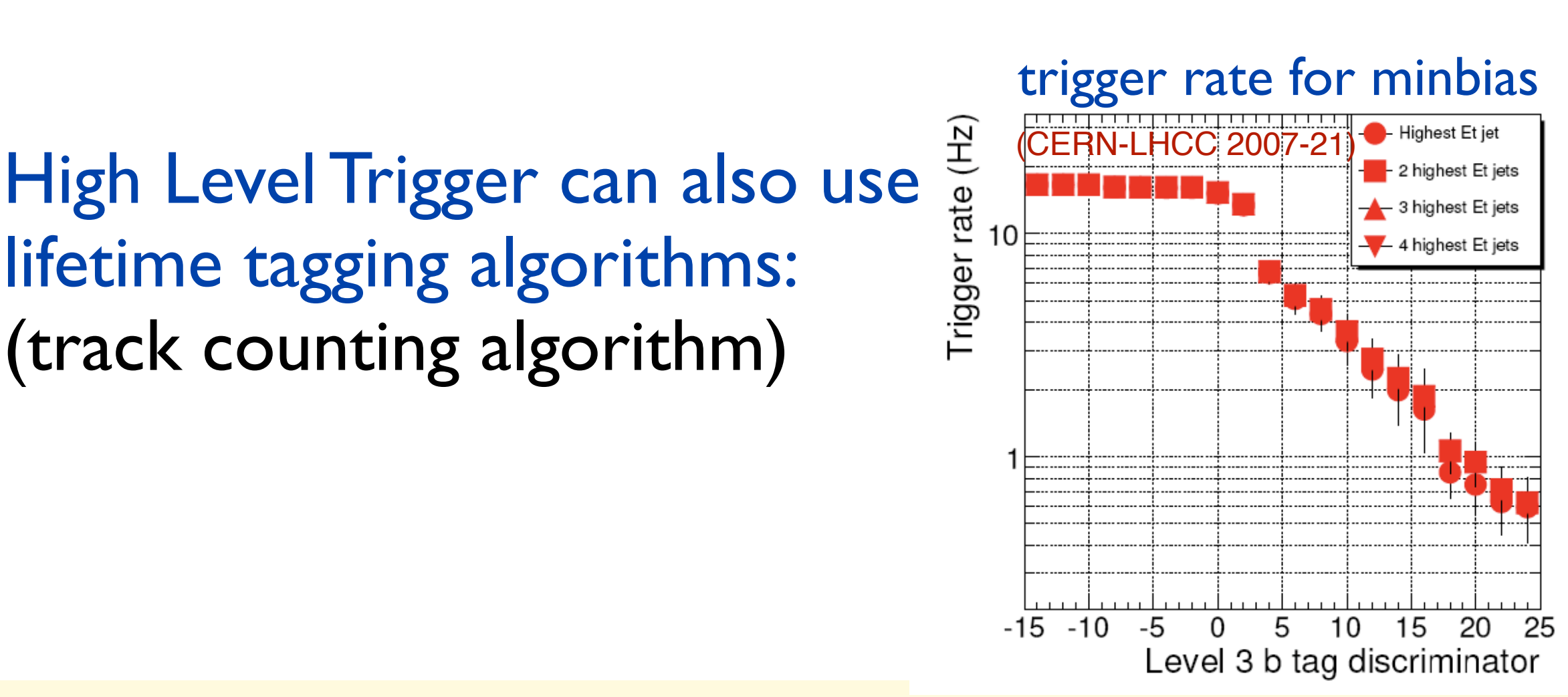
(track counting algorithm)

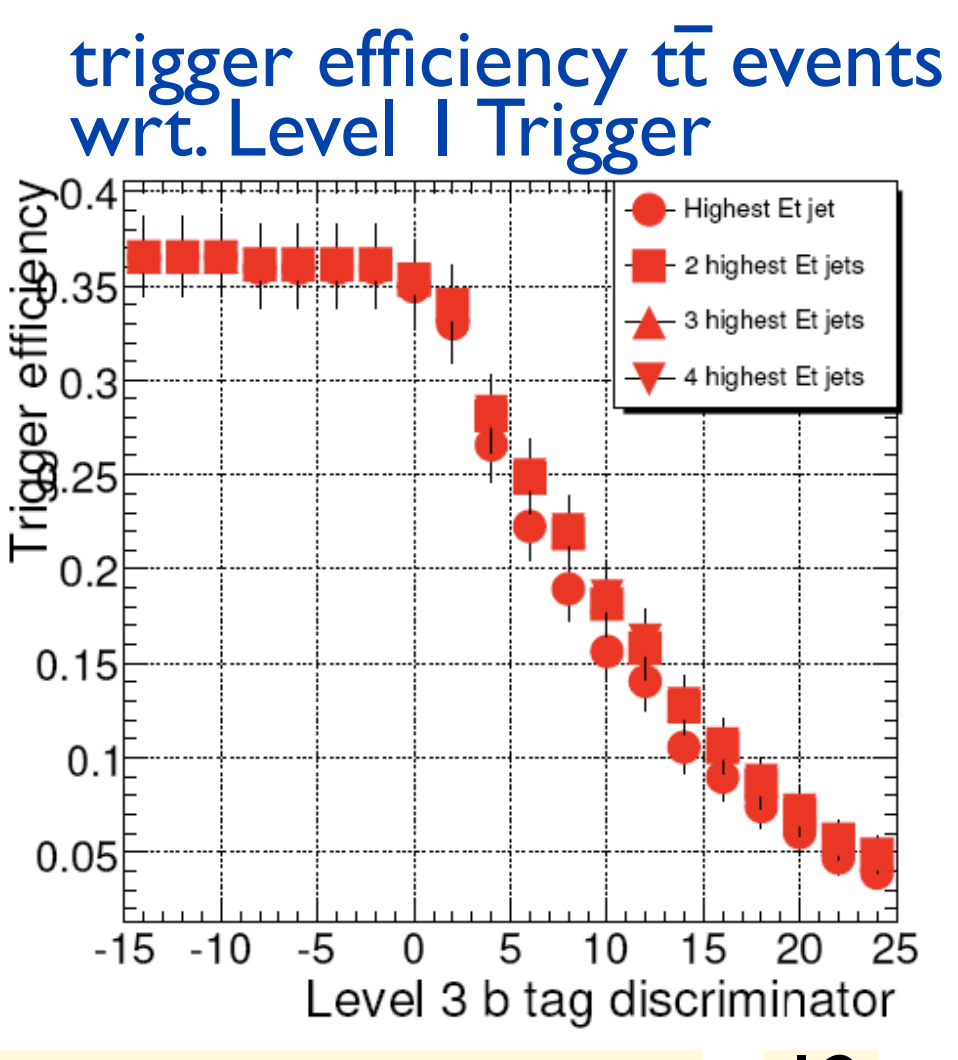

June 27 th, 2008 


\section{Measurement of Inclusive b-Production (II)}

\section{cross-section} measured differential in PT and eta.

$=>$ transverse energy and pseudo-rapidity of jets need to be well reconstructed
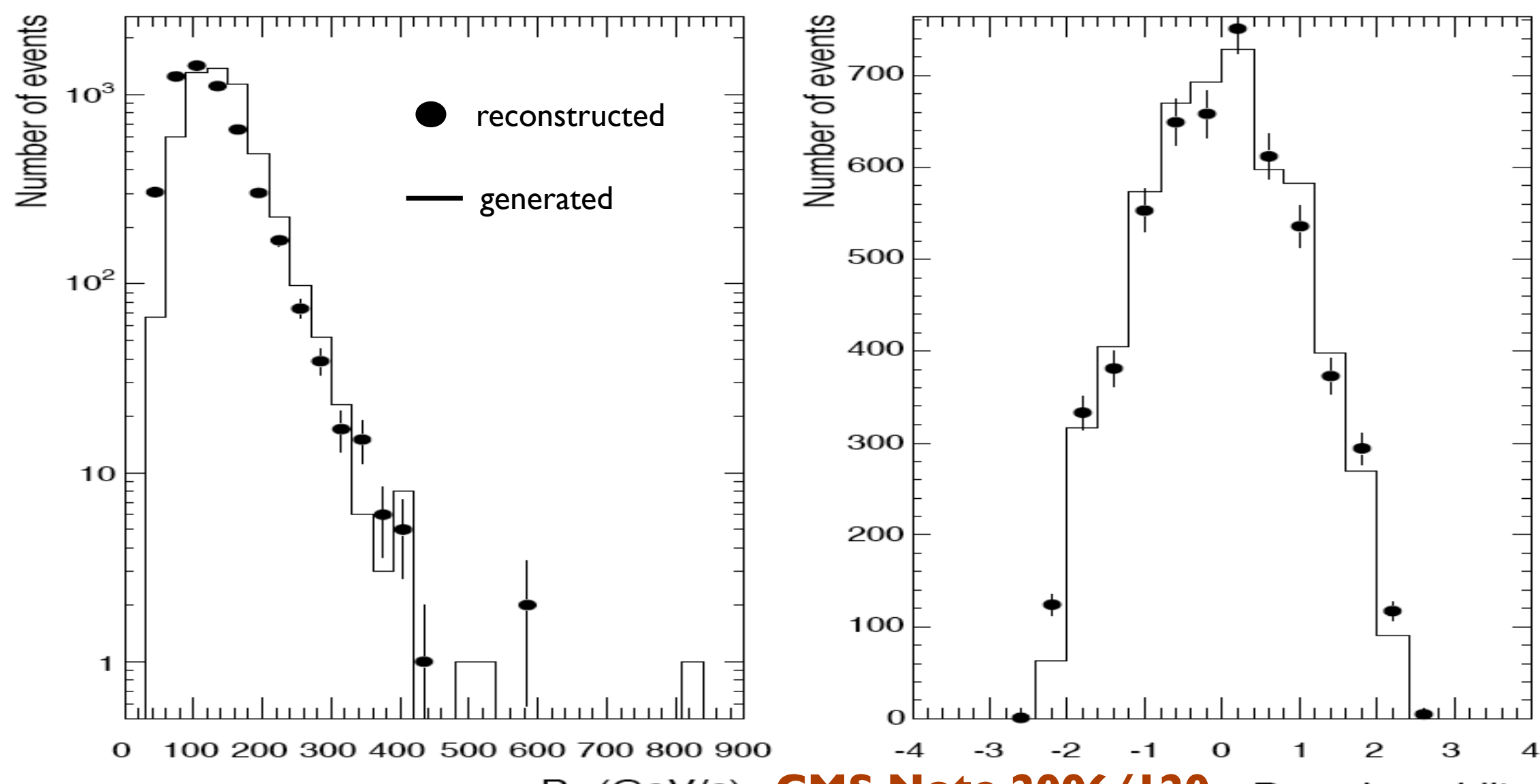

$\mathrm{P}_{\mathrm{T}}(\mathrm{GeV} / \mathrm{c})$

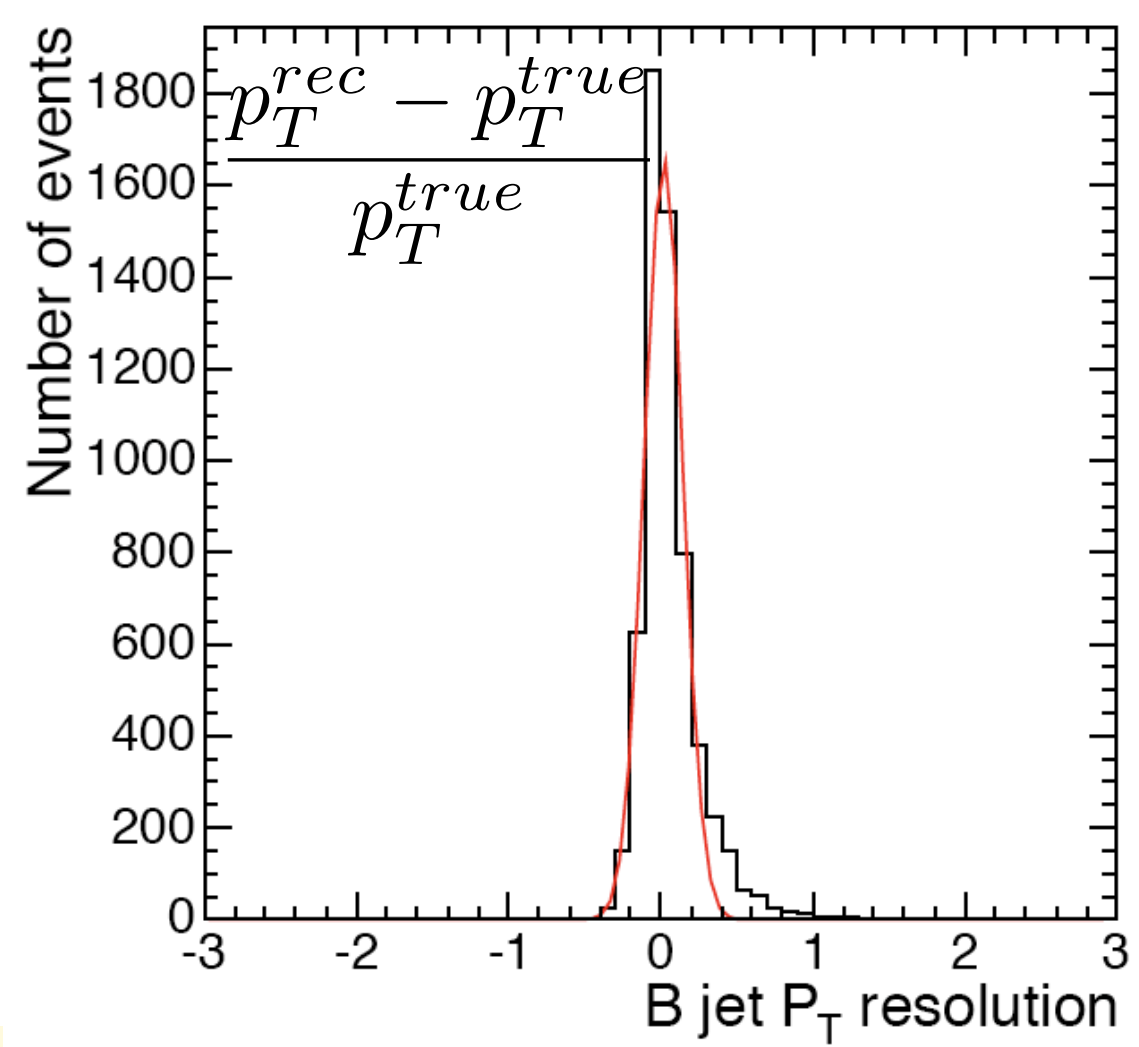

BEACH08, University of South Carolina

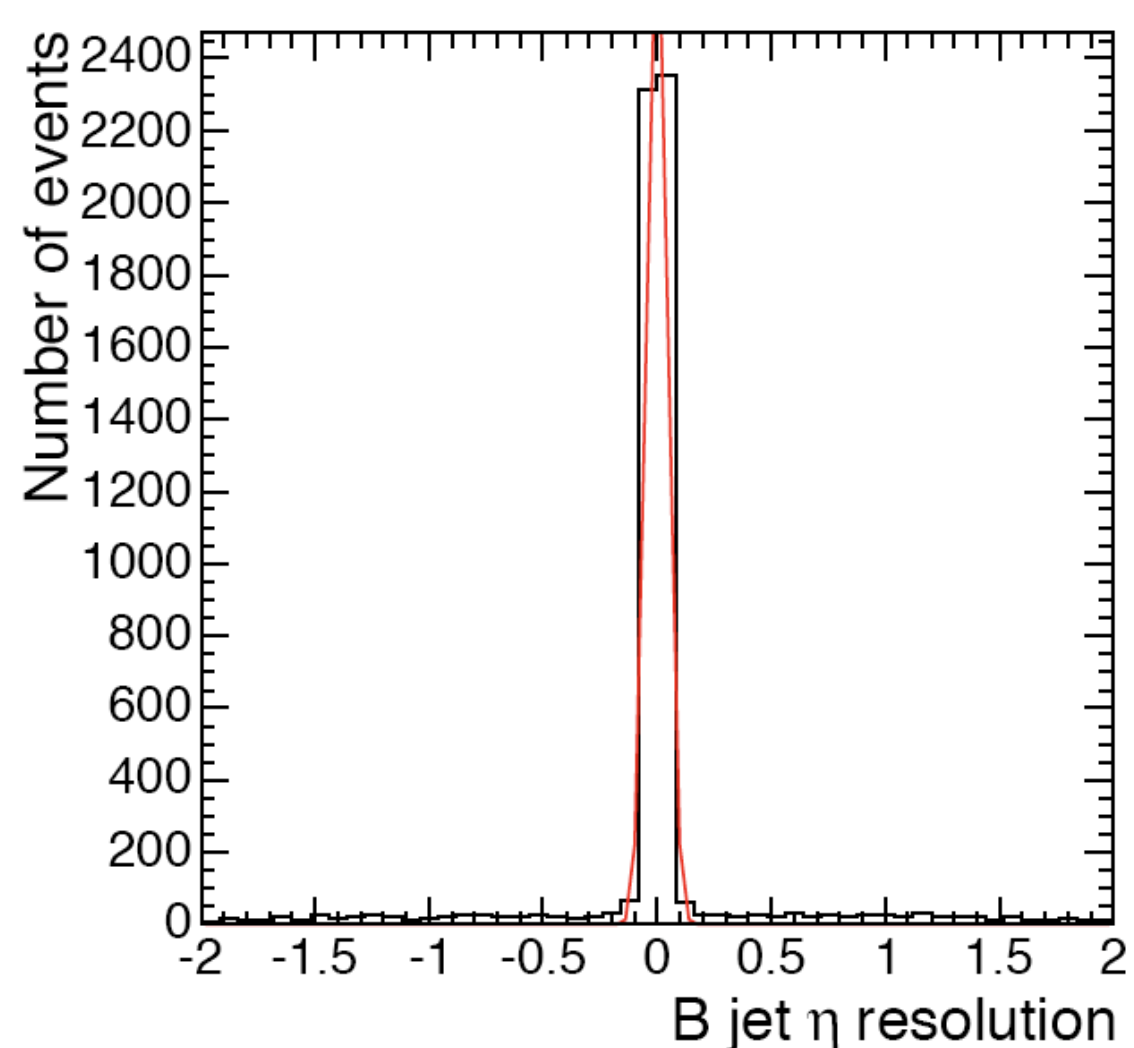

June 27 th, 2008 


\section{Measurement of Inclusive b-Production (III)}

to measure the cross section, one needs:

I. number of selected events

2. integrated luminosity

3. signal selection efficiency

4. signal purity (flavour fraction)

$=>$ measure $\mathrm{I}$. and 2 .

for 3. and 4. rely as few as possible on simulation.

tagging efficiencies and flavour fractions

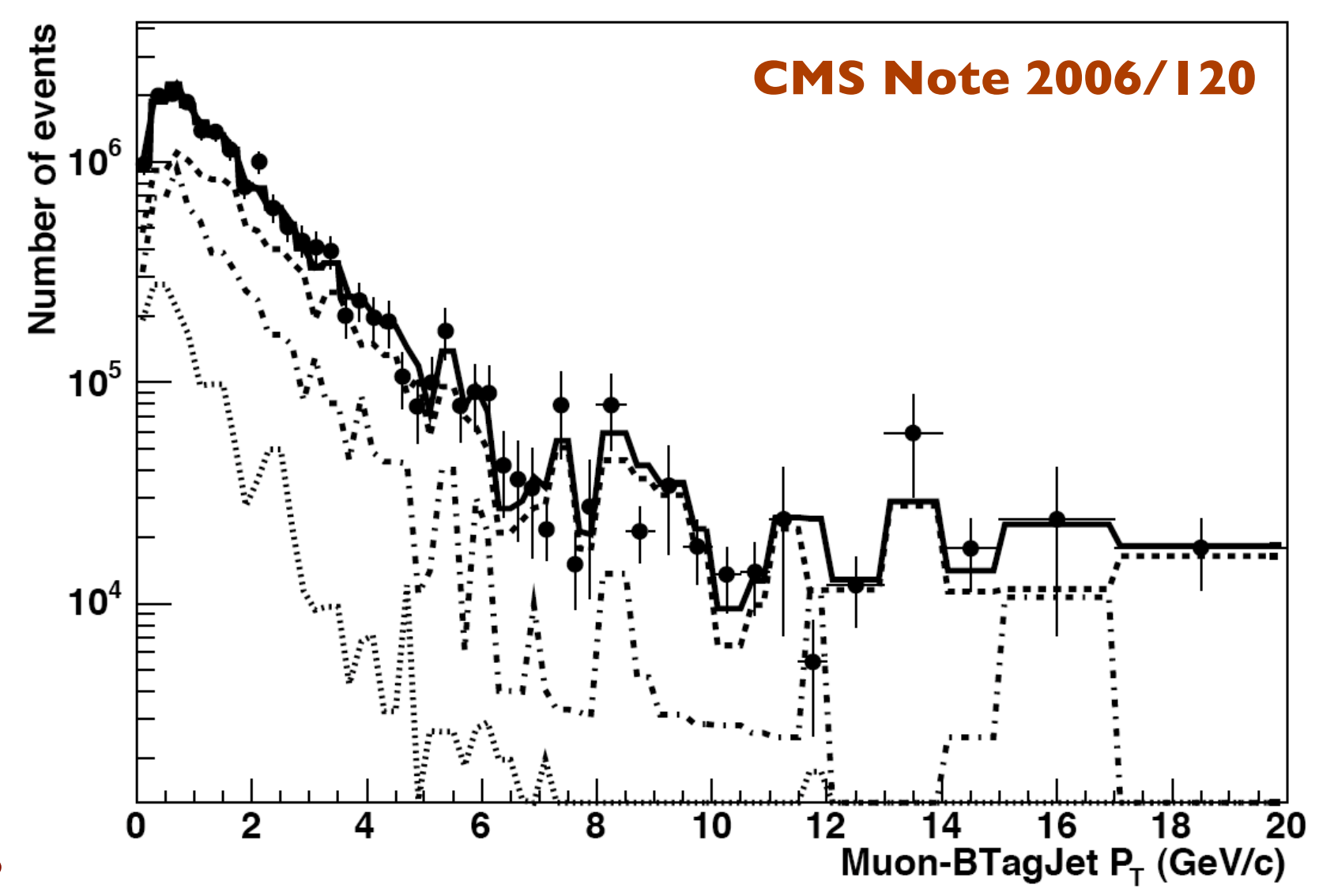
can also be measured, e.g. by fitting the relative transverse momentum of the muon to the jet

$=>$ can be done with a precision of about $10 \%$ for the b-content in $10 \mathrm{fb}^{-1}$ $=>$ contamination from $t \bar{t}$ events is $1 \%$ for $\mathrm{PT}<500 \mathrm{GeV}$ and $2.4 \%$ for $\mathrm{PT}>500 \mathrm{GeV}$ 


\section{Measurement of Inclusive b-Production (IV)}

systematic uncertainties taken into account:

- jet energy scale: $12 \%$ (depending on energy)

- model of detector response and lepton identification: $6 \%$

- b-tagging: $5 \%$

- luminosity: $5 \%$

- trigger: $<3 \%$

- fragmentation modeling

$=>$ systematic uncertainty of $10 \%$ uncertainty depending on transverse momentum for $1 \mathrm{Ofb}^{-1}$ : dominant below ITeV

$=>$ measurement limited to I.5TeV by statistical uncertainty 


\section{Measurement of b-Tagging Efficiencies}

-top quarks: select high purity b-jet sample based on top quark observables: mass resonances, leptons, angles, ...

-muons in jets: fitting relative рт of muon

-System8 method:

three uncorrelated identification criteria combined:

1. working point of (lifetime) algorithm

2. cut on muon PTrel

3. presence of second b-tagged-jet

(b-Quark pair production)

$=>$ get system of 8 linear equations with 8 unknowns (flavour content)

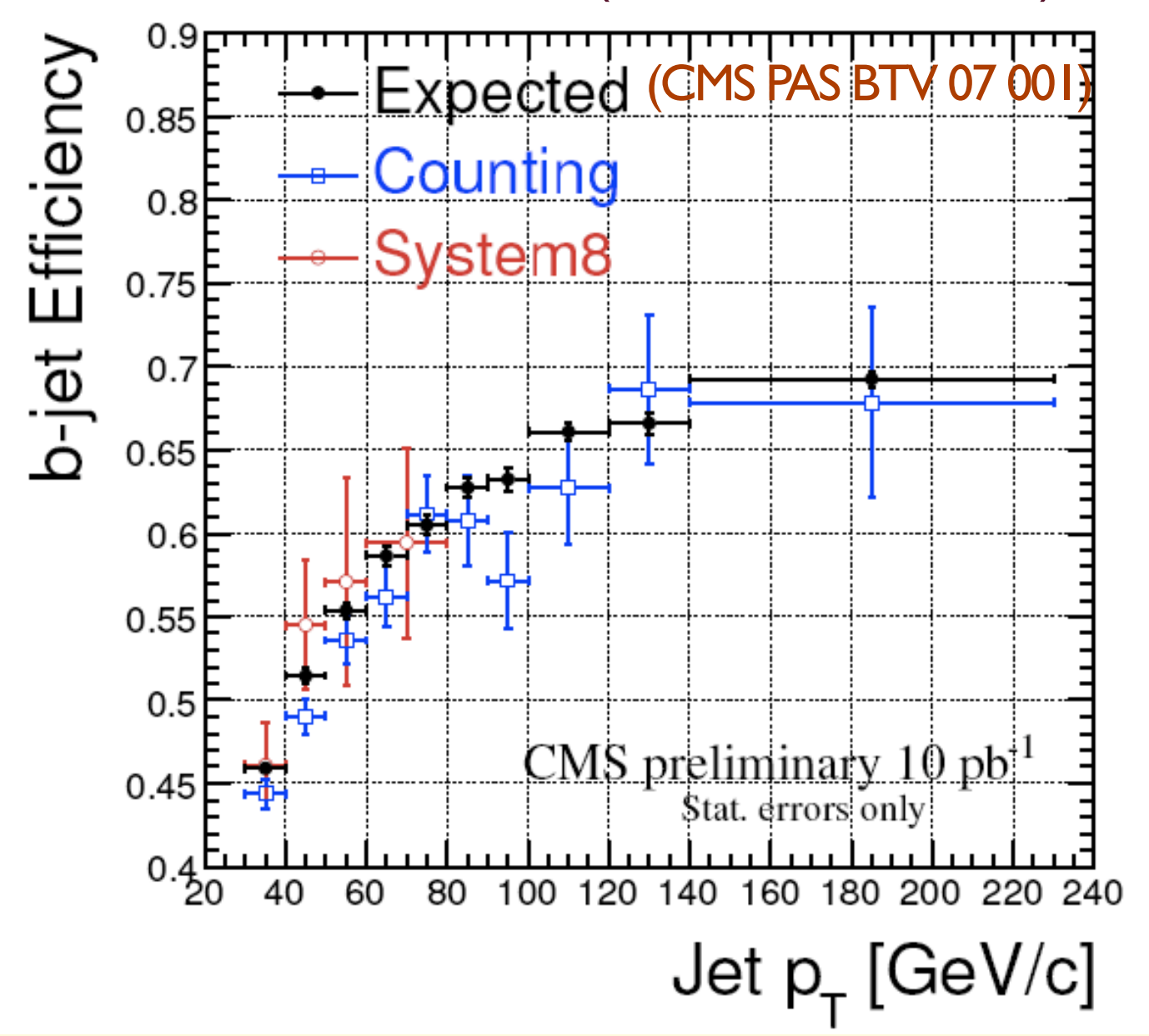

\section{-negative tags:}

using the negative tail in the IP distribution to estimate mistagging rates

light flavour jets show symmetric distribution in the ideal case

$=>$ negative tags allow measurement of mistagging rates with data

$\varepsilon_{\text {data }}^{\text {mistag }}=\varepsilon_{\text {data }}^{-} \cdot R_{\text {light }}$

can be done with $7 \%$ relative uncertainty for a mistag rate of $1 \%$

(CMS PAS BTV 07 002) 


\section{Conclusions}

- a large number of physics studies depend on efficient and clean identification of b-quark jets:

Higgs physics, BSM searches, top physics, ...

- knowledge of $b \bar{b}$ production is crucial for these searches

- CMS will be able to measure the differential $\mathrm{b} \overline{\mathrm{b}}$ production cross section up to $1.5 \mathrm{TeV}$

- the tools to do this all seem to be in place

- detector commissioning is making good progress $=>$ waiting for data... 